\title{
On the Failure of the Finite Model Property in some Fuzzy Description Logics
}

\author{
${ }^{1}$ Fernando Bobillo $\quad{ }^{2}$ Félix Bou \\ ${ }^{3}$ Umberto Straccia \\ 1 Department of Computer Science and Systems Engineering \\ University of Zaragoza, Spain \\ 2 Institut d'Investigació en Intelligència Artificial \\ Consejo Superior de Investigaciones Científicas (IIIA - CSIC), Bellaterra, Spain \\ 3 Istituto di Scienza e Tecnologie dell'Informazione \\ Consiglio Nazionale delle Ricerche (ISTI - CNR), Pisa, Italy
}

September 26, 2018

\begin{abstract}
Fuzzy Description Logics (DLs) are a family of logics which allow the representation of (and the reasoning with) structured knowledge affected by vagueness. Although most of the not very expressive crisp DLs, such as $\mathcal{A L C}$, enjoy the Finite Model Property (FMP), this is not the case once we move into the fuzzy case. In this paper we show that if we allow arbitrary knowledge bases, then the fuzzy DLs $\mathcal{A L C}$ under Eukasiewicz and Product fuzzy logics do not verify the FMP even if we restrict to witnessed models; in other words, finite satisfiability and witnessed satisfiability are different for arbitrary knowledge bases. The aim of this paper is to point out the failure of FMP because it affects several algorithms published in the literature for reasoning under fuzzy $\mathcal{A L C}$.
\end{abstract}

\section{Introduction}

Description Logics (DLs) [2] are a logical reconstruction of the so-called frame-based knowledge representation languages, with the aim of providing a simple well-established Tarski-style declarative semantics to capture the meaning of the most popular features of structured representation of knowledge. Nowadays, DLs have gained even more popularity due to their application in 
the context of the Semantic Web 4]. For example, the current standard language for specifying ontologies, the Web Ontology Language OWL is based on Description Logics.

It is very natural to extend DLs to the fuzzy case in order to manage fuzzy/vague/imprecise pieces of knowledge for which a clear and precise definition is not possible. For a good and recent survey on the advances in the field of fuzzy DLs, we refer the reader to [14]. One of the challenges of the research in this community is the fact that different families of fuzzy operators (or fuzzy logics) lead to fuzzy DLs with different properties.

In fuzzy logic, there are a lot of families of fuzzy operators (or fuzzy logics). Table 1 shows the connectives involved in what are considered the main four families. The most famous families correspond to the three basic continuous t-norms (i.e., Łukasiewicz, Gödel and Product [10]) together with an R-implication 1 . Besides these three, we also point out Zadeh's family corresponding to the operators originally proposed by Lotfi A. Zadeh in his seminal work 23: Gödel t-norm and t-conorm, Łukasiewicz negation and Kleene-Dienes implication.

Table 1: Popular families of fuzzy operators

\begin{tabular}{|l|l|l|l|l|}
\hline family & $\mathrm{t}$-norm $\alpha \otimes \beta$ & $\mathrm{t}$-conorm $\alpha \oplus \beta$ & negation $\ominus \alpha$ & implication $\alpha \Rightarrow \beta$ \\
\hline \hline Zadeh & $\min \{\alpha, \beta\}$ & $\max \{\alpha, \beta\}$ & $1-\alpha$ & $\max \{1-\alpha, \beta\}$ \\
\hline Lukasiewicz & $\max \{\alpha+\beta-1,0\}$ & $\min \{\alpha+\beta, 1\}$ & $1-\alpha$ & $\min \{1-\alpha+\beta, 1\}$ \\
\hline Product & $\alpha \cdot \beta$ & $\alpha+\beta-\alpha \cdot \beta$ & $\begin{cases}1, & \alpha=0 \\
0, & \alpha>0\end{cases}$ & $\begin{cases}1, & \alpha \leq \beta \\
\beta / \alpha, & \alpha>\beta\end{cases}$ \\
\hline Gödel & $\min \{\alpha, \beta\}$ & $\max \{\alpha, \beta\}$ & $\begin{cases}1, & \alpha=0 \\
0, & \alpha>0\end{cases}$ & $\begin{cases}1 & \alpha \leq \beta \\
\beta, & \alpha>\beta\end{cases}$ \\
\hline
\end{tabular}

The DL $\mathcal{A L C}$ 17] ( $\mathcal{A}$ ttributive $\mathcal{L}$ anguage with $\mathcal{C}$ omplements) is a very popular logic, usually considered as a standard. $\mathcal{A L C}$ is a notational variant of the multimodal logic $\mathbf{K}_{\mathbf{n}}$ (which essentially corresponds to not having a knowledge base) [16, and it is well known that $\mathbf{K}_{\mathbf{n}}$ verifies the Finite Model Property (FMP) (see [5, Proposition 2.15]). A logic verifies the FMP iff every satisfiable theory of the logic is also satisfiable by a finite model.

However, the situation is different in the fuzzy setting. It is also well known (see [11, Theorem 2] and [12, Theorem 82]) that in the context of fuzzy $\mathcal{A L C}$ the satisfiability problem for ABoxes (that is, the TBox is empty) in a finite model coincides with the satisfiability in the bigger class of witnessed models. This fact is used in [11 to prove that fuzzy $\mathcal{A L C}$ under Eukasiewicz enjoys the FMP since it is also known that first-order formulae satisfiable under Eukasiewicz semantics are always satisfiable in a witnessed model (see [13]). On the other hand, the FMP fails under Gödel and Product semantics even for ABoxes [11, Example 2].

\footnotetext{
${ }^{1}$ Every left-continuous t-norm $\otimes$ has associated a unique operation $\Rightarrow$ called the residuum of $\otimes$ (often called an R-implication) and defined as $\alpha \Rightarrow \beta=\sup \{\gamma \mid \alpha \otimes \gamma \leq \beta\}$.

${ }^{2}$ The result is formulated in a different framework (for example, without explicitly having a t-conorm), but the same proof given there works.
} 
The aim of this paper is to show that, contrary to what was thought 3 up to now, the previous result cannot be extended to arbitrary knowledge bases; that is, for arbitrary knowledge bases satisfiability in a finite model may not coincide with satisfiability in the class of witnessed models. In this paper we show that this happens at least in the cases of Eukasiewicz and Product semantics. For these two cases we provide knowledge bases that are satisfiable in a witnessed model but not in any finite model. In particular, it follows that FMP is false in Łukasiewicz when we allow arbitrary knowledge bases.

The main reason why we can build knowledge bases that are counterexamples to the FMP is the use of general concept inclusions in the TBox. Indeed, in Section 4 we show that if the TBox is unfoldable then satisfiability in a finite model coincides with satisfiability in a witnessed model.

The remainder of this document is as follows. Section 2 recalls the definition of fuzzy $\mathcal{A} \mathcal{L}$ s. Then, Section 3 shows that, under Eukasiewicz and Product semantics satisfiability of a knowledge base in a witnessed model does not coincide with satisfiability in a finite model. Finally, Section 4 discusses some consequences of this fact.

\section{Fuzzy Description Logics $\mathcal{A} \mathcal{L C}$}

The crisp DL $\mathcal{A L C}$ is one particular logic. On the other hand, its generalization to the fuzzy setting is not unique since it depends on which fuzzy operators are chosen (see Table 1). In this section, for every family of operators it is defined a particular $\mathcal{A L C}$ fuzzy logic based on the guidelines given in [19, 20. It is worth pointing out that all these fuzzy DLs share the same syntax, although their semantics are different.

Syntax Let $\mathbf{C}, \mathbf{R}$ and $\mathbf{I}$ be non-empty enumerable and pair-wise disjoint sets of concept names (denoted $A$ ), role names (denoted $R$ ) and individual names (denoted $a, b$ ). Concepts may be seen as unary predicates, while roles may be seen as binary predicates.

$\mathcal{A L C}$ (complex) concepts can be built according to the following syntax rule:

$$
C, D \rightarrow \top|\perp| A|C \sqcap D| C \sqcup D|\neg C| \forall R . C \mid \exists R . C
$$

There are no complex roles in $\mathcal{A L C}$, but only the atomic ones. Thus, the syntax here considered is essentially the same one than for crisp $\mathcal{A L C}$. Although it would be interesting to increase the expressive power adding an implication $C \Rightarrow D$ of concepts, we have adopted the convention to avoid it in order to present a weaker logic where still the negative results given in Section 3 hold.

An $\mathcal{A L C}$ fuzzy knowledge base (fuzzy $\mathrm{KB}) \mathcal{K}=\langle\mathcal{A}, \mathcal{T}\rangle$ consists of a fuzzy ABox $\mathcal{A}$ and a fuzzy TBox $\mathcal{T}$, where

\footnotetext{
${ }^{3}$ And implicitly used in several published algorithms (see Section 4).
} 
- a fuzzy $A B o x \mathcal{A}$ is a finite set of fuzzy concept assertion axioms of the form $\langle a: C \geq \alpha\rangle$ and $\langle a: C \leq \alpha\rangle$, and fuzzy role assertion axioms of the form 4 $\langle(a, b): R \geq \alpha\rangle$, where $a, b$ are individual names, $C$ is a concept, $R$ is a role and $\alpha \in[0,1]$ is a rational number.

- a fuzzy TBox $\mathcal{T}$ is a finite set of fuzzy general concept inclusion (GCI) axioms $\langle C \sqsubseteq D \geq \alpha\rangle$, where $C, D$ are concepts and $\alpha \in[0,1]$ is a rational number.

It is common to write $\tau$ as a shorthand for $\langle\tau \geq 1\rangle,\langle a: C=\alpha\rangle$ as an abbreviation of the pair of axioms $\langle a: C \geq \alpha\rangle$ and $\langle a: C \leq \alpha\rangle$, and the concept equivalence $C \equiv D$ as a shorthand of the two axioms $\langle C \sqsubseteq D \geq 1\rangle$ and $\langle D \sqsubseteq C \geq 1\rangle$.

For the sake of concrete illustration, let us introduce a couple of examples of fuzzy KBs. The second one will be used later as a counterexample to the FMP.

Example 2.1 The pair $\mathcal{K}_{1}=\left\langle\mathcal{A}_{1}, \mathcal{T}_{1}\right\rangle$ with $\mathcal{A}_{1}=\{\langle$ jim:YoungPerson $\geq 0.2\rangle$, $\langle(j$ im,mary $):$ likes $\geq 0.8\rangle\}$ and $\mathcal{T}_{1}=\{\langle$ Inn $\sqsubseteq$ Hotel $\geq 0.5\rangle\}$ is a fuzzy $K B$.

Example 2.2 $\mathcal{K}_{2}$ is the fuzzy $K B$ with the following axioms

(1) $(a: A)=0.5$

(2) $\top \sqsubseteq \exists R . \top$

(3) $(\forall R . A) \equiv(\exists R . A)$

(4) $A \equiv(\forall R . A) \sqcap(\forall R . A)$

Semantics In fuzzy DLs, concepts and roles are interpreted, respectively, as fuzzy subsets and fuzzy relations over an interpretation domain. However, the axioms of a fuzzy KB (i.e., the elements of its ABox or its TBox) are either satisfied (true) or unsatisfied (false) in an interpretation. Hence, the axioms behave as in the crisp case and they are not interpreted as a degree of truth in $[0,1]$.

Informally speaking, a fuzzy axiom $\langle\tau \geq \alpha\rangle$ in a ABox constrains the membership degree of $\tau$ to be at least $\alpha$. And the intended semantics of $\langle C \sqsubseteq D \geq \alpha\rangle$ states that all instances of concept $C$ are instances of concept $D$ to degree $\alpha$, i.e. the subsumption degree (to be clarified later) between $C$ and $D$ is at least $\alpha$. Next, we give precise definitions for the semantics just explained.

A fuzzy interpretation (or model) is a pair $\mathcal{I}=\left(\Delta^{\mathcal{I}},{ }^{\mathcal{I}}\right)$ consisting of a nonempty (crisp) set $\Delta^{\mathcal{I}}$ (the domain) and of a fuzzy interpretation function. ${ }^{\mathcal{I}}$ that assigns:

\footnotetext{
${ }^{4}$ The reader may be surprised about the fact that axioms of the form $\langle(a, b): R \leq \alpha\rangle$ are not considered. The reason to not accept this kind of statements is that in the case of crisp $\mathcal{A L C}$ in the ABox it is not allowed to use statements of the form $\langle(a, b): R \leq 0\rangle$ (remember that in $\mathcal{A L C}$ the negation of a role is not allowed). On the other hand, in crisp $\mathcal{A L C}$ it is indeed allowed to use $\langle a: C \leq 0\rangle$ since it corresponds to $\langle a: \neg C \geq 1\rangle$ (remember that the negation of a concept is allowed).
} 
Table 2: Fuzzy DL semantics for $\mathcal{A L C}$.

$$
\begin{aligned}
\perp^{\mathcal{I}}(x) & =0 \\
\top^{\mathcal{I}}(x) & =1 \\
(C \sqcap D)^{\mathcal{I}}(x) & =C^{\mathcal{I}}(x) \otimes D^{\mathcal{I}}(x) \\
(C \sqcup D)^{\mathcal{I}}(x) & =C^{\mathcal{I}}(x) \oplus D^{\mathcal{I}}(x) \\
(\neg C)^{\mathcal{I}}(x) & =\ominus C^{\mathcal{I}}(x) \\
(\forall R . C)^{\mathcal{I}}(x) & =\inf _{y \in \Delta^{\mathcal{I}}}\left(R^{\mathcal{I}}(x, y) \Rightarrow C^{\mathcal{I}}(y)\right) \\
(\exists R . C)^{\mathcal{I}}(x) & =\sup _{y \in \Delta^{\mathcal{I}}}\left(R^{\mathcal{I}}(x, y) \otimes C^{\mathcal{I}}(y)\right)
\end{aligned}
$$

1. to each atomic concept $A$ a function $A^{\mathcal{I}}: \Delta^{\mathcal{I}} \rightarrow[0,1]$,

2. to each role $R$ a function $R^{\mathcal{I}}: \Delta^{\mathcal{I}} \times \Delta^{\mathcal{I}} \rightarrow[0,1]$,

3. to each individual $a$ an element $a^{\mathcal{I}} \in \Delta^{\mathcal{I}}$.

The fuzzy interpretation function is extended to complex concepts as specified in the Table 2 (where $x, y \in \Delta^{\mathcal{I}}$ are elements of the domain, and as usual we use $\otimes, \oplus, \ominus$ and $\Rightarrow$ to denote respectively the t-norm, t-conorm, negation function and implication function of the corresponding family of fuzzy operators chosen). Hence, for every complex concept $C$ we get a function $C^{\mathcal{I}}: \Delta^{\mathcal{I}} \longrightarrow[0,1]$.

Note that $\langle a: C \leq \alpha\rangle$ is equivalent to $\langle a: \neg C \geq 1-\alpha\rangle$ under Eukasiewicz negation. Hence, in Łukasiewicz it is not necessary to explicitly consider fuzzy concept assertions of the form $\langle a: C \leq \alpha\rangle$.

The (crisp) satisfiability of axioms in a fuzzy $K B$ is then defined by the following conditions:

1. $\mathcal{I}$ satisfies an axiom $\langle a: C \geq \alpha\rangle$ in case that $C^{\mathcal{I}}\left(a^{\mathcal{I}}\right) \geq \alpha$,

2. $\mathcal{I}$ satisfies an axiom $\langle a: C \leq \alpha\rangle$ in case that $C^{\mathcal{I}}\left(a^{\mathcal{I}}\right) \leq \alpha$,

3. $\mathcal{I}$ satisfies an axiom $\langle(a, b): R \geq \alpha\rangle$ in case that $R^{\mathcal{I}}\left(a^{\mathcal{I}}, b^{\mathcal{I}}\right) \geq \alpha$,

4. $\mathcal{I}$ satisfies an axiom $\langle C \sqsubseteq D \geq \alpha\rangle$ in case that $(C \sqsubseteq D)^{\mathcal{I}} \geq \alpha$ where $(C \sqsubseteq D)^{\mathcal{I}}=\inf _{x \in \Delta^{\mathcal{I}}}\left(C^{\mathcal{I}}(x) \Rightarrow D^{\mathcal{I}}(x)\right)$.

It is interesting to point out that the satisfaction of a GCI of the form $\langle C \sqsubseteq D \geq 1\rangle$ is exactly the requirement that $\forall x \in \Delta^{\mathcal{I}}, C^{\mathcal{I}}(x) \leq D^{\mathcal{I}}(x)$ (i.e., Zadeh's set inclusion); hence, in this particular case for the satisfaction it only matters the partial order and not the exact value of the implication $\Rightarrow$.

As it is expected we will say that a fuzzy interpretation $\mathcal{I}$ satisfies a $\mathrm{KB} \mathcal{K}$ in case that it satisfies all axioms in $\mathcal{K}$. And it is said that a fuzzy $\mathrm{KB} \mathcal{K}$ is satisfiable iff there exist a fuzzy interpretation $\mathcal{I}$ satisfying every axiom in $\mathcal{K}$.

As it is said in the introduction, here we mainly focus on witnessed models and compare them to finite models. This notion (see [11) corresponds to the restriction to the DL language of the notion of witnessed model introduced, in 
the context of the first-order language, by Hájek in [13]. A fuzzy interpretation $\mathcal{I}$ is said to be witnessed iff it holds that for every complex concepts $C, D$, every role $R$, and every $x \in \Delta^{\mathcal{I}}$ there is some

1. $y \in \Delta^{\mathcal{I}}$ such that $(\exists R . C)^{\mathcal{I}}(x)=R^{\mathcal{I}}(x, y) \otimes C^{\mathcal{I}}(y)$.

2. $y \in \Delta^{\mathcal{I}}$ such that $(\forall R . C)^{\mathcal{I}}(x)=R^{\mathcal{I}}(x, y) \Rightarrow C^{\mathcal{I}}(y)$.

3. $y \in \Delta^{\mathcal{I}}$ such that $(C \sqsubseteq D)^{\mathcal{I}}=C^{\mathcal{I}}(y) \Rightarrow D^{\mathcal{I}}(y)$.

The idea behind this definition is that in a witnessed interpretation all arbitrary infima and suprema needed in order to compute $C^{\mathcal{I}}$ and $(C \sqsubseteq D)^{\mathcal{I}}$ are indeed minima and maxima. It is obvious that all finite fuzzy interpretations (this means that $\Delta^{\mathcal{I}}$ is a finite set) are indeed witnessed, but the opposite is not true. A fuzzy $\mathrm{KB} \mathcal{K}$ is said to be satisfiable in a witnessed interpretation iff there exist a witnessed fuzzy interpretation $\mathcal{I}$ satisfying every axiom in $\mathcal{K}$.

\section{$3 \quad$ Infinite Models in Fuzzy $\mathcal{A L C}$}

In this section we prove that there are fuzzy KBs that are not finitely satisfiable while are satisfiable in a witnessed interpretation. We will prove this statement for two cases: the Eukasiewicz and the Product cases, and in both cases we will use the same fuzzy $\mathrm{KB} \mathcal{K}_{2}$ defined in Example 2.2.

First of all we focus in the Lukasiewicz case. It is worth pointing out that under Eukasiewicz semantics the first axiom in $\mathcal{K}_{2}$ can be rewritten as

$$
\langle a:((A \sqcup A) \sqcap \neg(A \sqcap A)) \geq 1\rangle,
$$

and so it is simply using as a bound the crisp value 1 .

Proposition 3.1 Let $\mathcal{I}$ be a witnessed model of $\mathcal{K}_{2}$ under Lukasiewicz fuzzy logic. Then, for every natural number $n$ there are individuals $b_{1}, b_{2}, b_{3}, \ldots, b_{n}$ such that $0.5=A^{\mathcal{I}}\left(b_{1}\right)<A^{\mathcal{I}}\left(b_{2}\right)<A^{\mathcal{I}}\left(b_{3}\right)<\cdots<A^{\mathcal{I}}\left(b_{n}\right)<1$.

Proof: For the case $n=1$ this is trivial using axiom (1) in the fuzzy KB.

Now let us assume that there are individuals $b_{1}, b_{2}, b_{3}, \ldots, b_{n}$ such that $0.5=$ $A^{\mathcal{I}}\left(b_{1}\right)<A^{\mathcal{I}}\left(b_{2}\right)<A^{\mathcal{I}}\left(b_{3}\right)<\cdots<A^{\mathcal{I}}\left(b_{n}\right)<1$. We want to check that there is an individual $b_{n+1}$ such that $A^{\mathcal{I}}\left(b_{n}\right)<A^{\mathcal{I}}\left(b_{n+1}\right)<1$.

Using axiom (2) we know that $(\exists R . \top)^{\mathcal{I}}\left(b_{n}\right)=1$. Since the model is witnessed, there is a 1 -successor of $b_{n}$ that we call $b$, i.e., $R^{\mathcal{I}}\left(b_{n}, b\right)=1$. It is obvious that $(\forall R \cdot A)^{\mathcal{I}}\left(b_{n}\right) \leq 1 \Rightarrow A^{\mathcal{I}}(b)=A^{\mathcal{I}}(b)$ and that $A^{\mathcal{I}}(b)=1 \otimes A^{\mathcal{I}}(b) \leq$ $(\exists R \cdot A)^{\mathcal{I}}\left(b_{n}\right)$. Using axiom $(3)$ we get that $(\forall R . A)^{\mathcal{I}}\left(b_{n}\right)=A^{\mathcal{I}}(b)=(\exists R \cdot A)^{\mathcal{I}}\left(b_{n}\right)$. Therefore, axiom (4) is saying that $A^{\mathcal{I}}\left(b_{n}\right)=(\forall R \cdot A)^{\mathcal{I}}\left(b_{n}\right) \otimes(\forall R \cdot A)^{\mathcal{I}}\left(b_{n}\right)=$ $A^{\mathcal{I}}(b) \otimes A^{\mathcal{I}}(b)$. Using Eukasiewicz t-norm, it follows that $A^{\mathcal{I}}\left(b_{n}\right)=A^{\mathcal{I}}(b)+$ $A^{\mathcal{I}}(b)-1$, and hence $A^{\mathcal{I}}(b)=\left(A^{\mathcal{I}}\left(b_{n}\right)+1\right) / 2$. Thus, it is clear that $A\left(b_{n}\right)<$ $A(b)<1$. Hence, we can define $b_{n+1}$ as $b$ to finish the proof.

Q.E.D.

Corollary 3.2 There is no finite model for $\mathcal{K}_{2}$ under Eukasiewicz fuzzy logic. 
Theorem 3.3 $\mathcal{K}_{2}$ is, under Eukasiewicz fuzzy logic, satisfiable by a witnessed model but not by a finite model.

Proof: By the previous corollary it is enough to give a witnessed model of $\mathcal{K}_{2}$. One witnessed model of this fuzzy KB is the model $\mathcal{I}$ defined by

- $\Delta^{\mathcal{I}}=\{1,2,3, \ldots\} \cup\{\infty\}$,

- $R^{\mathcal{I}}$ is the crisp relation $\{(i, i+1): i=1,2,3, \ldots\} \cup\{(\infty, \infty)\}$,

- $A^{\mathcal{I}}(\infty)=1$ and $A^{\mathcal{I}}(i)=\left(2^{i}-1\right) / 2^{i}$ for every $i=1,2,3, \ldots$

- $a^{\mathcal{I}}=1$.

The fact that this model $\mathcal{I}$ satisfies $\mathcal{K}_{2}$ can be easily checked by the reader, and the same for the first two conditions in the definition of witnessed model. On the other hand, the last condition in the definition of witnessed model is subtler, and we give here a proof. First of all we notice the following claim.

Claim 3.4 For every (complex) concept $C$ and every $\varepsilon>0$, it holds one (and only one) of the following two conditions:

$\boldsymbol{C o n d}_{\mathbf{1}}(\boldsymbol{C}, \boldsymbol{\varepsilon}): C^{\mathcal{I}}(\infty)=0$, and there is some $n \in \mathbb{N}$ such that $\inf \left\{C^{\mathcal{I}}(i): i \in\right.$ $\mathbb{N}, i \geq n\}=0$ and $\sup \left\{C^{\mathcal{I}}(i): i \in \mathbb{N}, i \geq n\right\} \leq \varepsilon$.

$\boldsymbol{C o n d}_{\mathbf{2}}(\boldsymbol{C}, \boldsymbol{\varepsilon}): C^{\mathcal{I}}(\infty)=1$, and there is some $n \in \mathbb{N}$ such that $\sup \left\{C^{\mathcal{I}}(i): i \in\right.$ $\mathbb{N}, i \geq n\}=1$ and $\inf \left\{C^{\mathcal{I}}(i): i \in \mathbb{N}, i \geq n\right\} \geq 1-\varepsilon$.

Proof of Claim: First of all, we notice that a trivial induction proves that $C^{\mathcal{I}}(\infty) \in\{0,1\}$ for every concept $C$. Next we prove the claim by induction on the length of the concept $C$. The cases of $T$ and an atomic concept $A$ are trivial. Due to the interdefinability between connectives in the Eukasiewicz case, it is enough to study the connectives $\neg, \sqcap$ and $\forall R$.C.

- The case of a concept $\neg C$ follows from the continuity of the Eukasiewicz negation; we remind the reader that continuity tells us that the function commutes with infima and suprema.

- Let us consider the case of a concept $C \sqcap D$. If both $C$ and $D$ satisfy the second condition, then it is enough to see that $\operatorname{Cond}_{2}(C, \varepsilon / 2)$ together with $\operatorname{Cond}_{2}(D, \varepsilon / 2)$ implies $\operatorname{Cond}_{2}(C \sqcap D, \varepsilon)$; and this fact is a consequence of $\sup \left\{(C \sqcap D)^{\mathcal{I}}(i): i \in \mathbb{N}, i \geq n\right\}=\sup \left\{C^{\mathcal{I}}(i): i \in \mathbb{N}, i \geq n\right\} \otimes \sup \left\{D^{\mathcal{I}}(i):\right.$ $i \in \mathbb{N}, i \geq n\}=1 \otimes 1=1$ (by the continuity of $\otimes$ ) and $\left(1-\frac{\varepsilon}{2}\right) \otimes\left(1-\frac{\varepsilon}{2}\right) \geq$ $1-\varepsilon$. Let us consider now the case that at least one of the two concepts satisfies the first condition. Without loss of generality, we can assume that $\operatorname{Cond}_{1}(C, \varepsilon)$ holds. From this assumption it follows $\operatorname{Cond}_{1}(C \sqcap D, \varepsilon)$ because $(C \sqcap D)^{\mathcal{I}}(i) \leq C^{\mathcal{I}}(i)$ for every $i$.

- The case of a concept $\forall R$.C follows from the fact that for every $i \in \mathbb{N}$, $(\forall R . C)^{\mathcal{I}}(i)=C^{\mathcal{I}}(i+1)$. 
This finishes the proof of the claim.

Q.E.D.

Let us now prove the third requirement in the definition of a witnessed interpretation. Hence, we consider two concepts $C$ and $D$. Using the previous claim for the concept $\neg C \sqcup D$ we are in one of the following two cases.

- Case $(\neg C \sqcup D)^{\mathcal{I}}(\infty)=0$. In this case it is trivial that $(C \sqsubseteq D)^{\mathcal{I}}=0=$ $C^{\mathcal{I}}(\infty) \Rightarrow D^{\mathcal{I}}(\infty)$.

- Case $(\neg C \sqcup D)^{\mathcal{I}}(\infty)=1$ and for every $\varepsilon>0$ there is some $n \in \mathbb{N}$ such that $\sup \left\{(\neg C \sqcup D)^{\mathcal{I}}(i): i \in \mathbb{N}, i \geq n\right\}=1$ and $\inf \left\{(\neg C \sqcup D)^{\mathcal{I}}(i): i \in \mathbb{N}, i \geq\right.$ $n\} \geq 1-\varepsilon$. Without loss of generality we can assume that there is some $m \in \mathbb{N}$ such that $(\neg C \sqcup D)^{\mathcal{I}}(m)<1$, because if not, then $(C \sqsubseteq D)^{\mathcal{I}}=1$ and hence $(C \sqsubseteq D)^{\mathcal{I}}=C^{\mathcal{I}}(i) \Rightarrow D^{\mathcal{I}}(i)$ for every $i \in I$. Let us consider $a:=(\neg C \sqcup D)^{\overline{\mathcal{I}}}(m)$ and $\varepsilon:=1-a$. Thus, using the claim we know that there is some $n \in \mathbb{N}$ such that $\sup \left\{(\neg C \sqcup D)^{\mathcal{I}}(i): i \in \mathbb{N}, i \geq n\right\}=1$ and $\inf \left\{(\neg C \sqcup D)^{\mathcal{I}}(i): i \in \mathbb{N}, i \geq n\right\} \geq a$. Hence,

$$
(C \sqsubseteq D)^{\mathcal{I}}=\min \left(\left\{(\neg C \sqcup D)^{\mathcal{I}}(m)\right\} \cup\left\{(\neg C \sqcup D)^{\mathcal{I}}(i): i \leq n\right\}\right) .
$$

As $(C \sqsubseteq D)^{\mathcal{I}}$ is the minimum of a finite set the proof is finished. Q.E.D.

Next we consider the case of Product logic. The following proposition can be proved by essentially the same argument as for Proposition 3.1] so we skip the proof.

Proposition 3.5 Let us a consider a witnessed model $\mathcal{I}$ of $\mathcal{K}_{2}$ under Product fuzzy logic. Then, for every natural number $n$ there are individuals $b_{1}, b_{2}, b_{3}, \ldots, b_{n}$ such that $0.5=A^{\mathcal{I}}\left(b_{1}\right)<A^{\mathcal{I}}\left(b_{2}\right)<A^{\mathcal{I}}\left(b_{3}\right)<\cdots<A^{\mathcal{I}}\left(b_{n}\right)<1$.

Corollary 3.6 There is no finite model for $\mathcal{K}_{2}$ under Product fuzzy logic.

Theorem 3.7 $\mathcal{K}_{2}$ is, under Product fuzzy logic, satisfiable by a witnessed model but not by a finite model.

Proof: It is enough to give a witnessed model of $\mathcal{K}_{2}$. One witnessed model of this fuzzy $\mathrm{KB}$ is the model $\mathcal{I}$ defined by

- $\Delta^{\mathcal{I}}=\{1,2,3, \ldots\}$,

- $R^{\mathcal{I}}$ is the crisp relation $\{(i, i+1): i=1,2,3, \ldots\}$,

- $A^{\mathcal{I}}(i)=\sqrt[2^{i-1}]{\left(\frac{1}{2}\right)}$ for every $i=1,2,3, \ldots$,

- $a^{\mathcal{I}}=1$.

The fact that this model $\mathcal{I}$ satisfies $\mathcal{K}_{2}$ can be easily checked by the reader, and the same for the first two conditions in the definition of witnessed model. The rest of this proof is devoted to prove the third condition. 
Claim 3.8 For all (complex) concepts $C$, one (and only one) of the following conditions holds:

- $0=C^{\mathcal{I}}(i)$ for every $i \in \mathbb{N}$,

- $0<C^{\mathcal{I}}(1) \leq C^{\mathcal{I}}(2) \leq C^{\mathcal{I}}(3) \leq \ldots$ and $\sup \left\{C^{\mathcal{I}}(i): i \in \mathbb{N}\right\}=1$.

Proof of Claim: This can be straightforwardly proved by induction on the length of the concept $C$ using how the fuzzy operators involved in the product case are defined 5 .

- The case of a concept $\neg C$ is a trivial consequence of the fact that Product negation is the one given in Table 1 (usually called strict negation).

- The cases of concepts built with $\sqcap$ and $\sqcup$ follow from the monotonicity of the functions $\otimes$ and $\oplus$.

- The cases of concepts built with $\forall R$. and $\exists R$. follow from the fact that for every $i \in \mathbb{N},(\forall R . C)^{\mathcal{I}}(i)=C^{\mathcal{I}}(i+1)=(\exists R \cdot C)^{\mathcal{I}}(i)$.

This finishes the proof of the claim.

Q.E.D.

Now it is time to use this previous claim in order to check that for every pair of concepts $C$ and $D$ there is some $i \in \mathbb{N}$ such that $(C \sqsubseteq D)^{\mathcal{I}}=C^{\mathcal{I}}(i) \Rightarrow D^{\mathcal{I}}(i)$. By the previous property we can distinguish three different cases, which cover all possibilities.

- Firstly, we consider the case where $0=C^{\mathcal{I}}(i)$ for every $i \in \mathbb{N}$. Then, it is trivial that $(C \sqsubseteq D)^{\mathcal{I}}=\inf \left\{0 \Rightarrow D^{\mathcal{I}}(i): i \in \mathbb{N}\right\}=1=C^{\mathcal{I}}(i) \Rightarrow D^{\mathcal{I}}(i)$ for every $i \in \mathbb{N}$.

- Secondly, we consider the case where $0 \neq C^{\mathcal{I}}(i)$ and $0=D^{\mathcal{I}}(i)$ for every $i \in \mathbb{N}$. Then, it is trivial that $(C \sqsubseteq D)^{\mathcal{I}}=\inf \left\{C^{\mathcal{I}}(i) \Rightarrow 0: i \in \mathbb{N}\right\}=0=$ $C^{\mathcal{I}}(i) \Rightarrow D^{\mathcal{I}}(i)$ for every $i \in \mathbb{N}$.

- Lastly, we consider the case where $0<C^{\mathcal{I}}(1) \leq C^{\mathcal{I}}(2) \leq C^{\mathcal{I}}(3) \leq \ldots$, $\sup \left\{C^{\mathcal{I}}(i): i \in \mathbb{N}\right\}=1,0<D^{\mathcal{I}}(1) \leq D^{\mathcal{I}}(2) \leq D^{\mathcal{I}}(3) \leq \ldots$ and $\sup \left\{D^{\mathcal{I}}(i): i \in \mathbb{N}\right\}=1$. In order to finish this proof it suffices to consider the case that $(C \sqsubseteq D)^{\mathcal{I}} \neq 1$, because if $(C \sqsubseteq D)^{\mathcal{I}}=1$ then it is clear that $(C \sqsubseteq D)^{\mathcal{I}}=C^{\mathcal{I}}(i) \Rightarrow D^{\mathcal{I}}(i)$ for every $i \in I$. Hence, let us assume that $(C \sqsubseteq D)^{\mathcal{I}} \neq 1$. Thus, there is some $i_{0} \in \mathbb{N}$ such that $\left(C^{\mathcal{I}}\left(i_{0}\right) \Rightarrow D^{\mathcal{I}}\left(i_{0}\right)\right)=\frac{D^{\mathcal{I}}\left(i_{0}\right)}{C^{\mathcal{I}}\left(i_{0}\right)}<1$. On the other hand, by continuity we know that

$$
\begin{gathered}
\lim _{i \rightarrow \infty} \min \left\{\frac{C^{\mathcal{I}}(i)}{D^{\mathcal{I}}(i)}, \frac{D^{\mathcal{I}}(i)}{C^{\mathcal{I}}(i)}\right\}=\min \left\{\frac{\lim _{i \rightarrow \infty} C^{\mathcal{I}}(i)}{\lim _{i \rightarrow \infty} D^{\mathcal{I}}(i)}, \frac{\lim _{i \rightarrow \infty} D^{\mathcal{I}}(i)}{\lim _{i \rightarrow \infty} C^{\mathcal{I}}(i)}\right\}= \\
=\min \left\{\frac{\sup _{i \in \mathbb{N}} C^{\mathcal{I}}(i)}{\sup _{i \in \mathbb{N}} D^{\mathcal{I}}(i)}, \frac{\sup _{i \in \mathbb{N}} D^{\mathcal{I}}(i)}{\sup _{i \in \mathbb{N}} C^{\mathcal{I}}(i)}\right\}=1
\end{gathered}
$$

\footnotetext{
${ }^{5}$ Here, as opposed to the Lukasiewicz case, the fact that the implication operator $\Rightarrow$ is not involved in the construction of complex $\mathcal{A L C}$ concepts is crucial.
} 
Therefore, as a consequence of the fact that for every $i \in \mathbb{N}$,

$$
\min \left\{\frac{C^{\mathcal{I}}(i)}{D^{\mathcal{I}}(i)}, \frac{D^{\mathcal{I}}(i)}{C^{\mathcal{I}}(i)}\right\} \leq C^{\mathcal{I}}(i) \Rightarrow D^{\mathcal{I}}(i) \leq 1,
$$

it follows that $\lim _{i \rightarrow \infty}\left(C^{\mathcal{I}}(i) \Rightarrow D^{\mathcal{I}}(i)\right)=1$. Thus, we know that the set $I=\left\{i \in \mathbb{N}: C^{\mathcal{I}}(i) \Rightarrow D^{\mathcal{I}}(i) \leq C^{\mathcal{I}}\left(i_{0}\right) \Rightarrow D^{\mathcal{I}}\left(i_{0}\right)\right\}$ is finite. Since $I$ is finite it is obvious that there is some $i \in I$ such that $(C \sqsubseteq D)^{\mathcal{I}}=C^{\mathcal{I}}(i) \Rightarrow$ $D^{\mathcal{I}}(i)$.

Q.E.D.

\section{Discussion}

The aim of this section is to discuss some interesting remarks of the failure of the FMP exposed in Theorems 3.3 and 3.7

The first remark is that the failure of the FMP is essentially a consequence of the fact that the TBox is cyclic. As we will see, in the case of Eukasiewicz this situation does not happen if we consider only unfoldable KBs.

A TBox $\mathcal{T}$ is acyclic iff it verifies the following three constraints:

1. Every axiom in $\mathcal{T}$ is either of the form $\langle A \sqsubseteq C \geq \alpha\rangle$ or $A \equiv C$, where $A$ is an atomic concept 6

2. There is no concept $A$ such that it appears more than once on the left hand side of some axiom in $\mathcal{T}$.

3. No cyclic definitions are present in $\mathcal{T}$. We will say that $A$ directly uses primitive concept $B$ in $\mathcal{T}$, if there is some axiom $\tau \in \mathcal{T}$ such that $A$ is on the left hand side of $\tau$ and $B$ occurs in the right hand side of $\tau$. Let uses be the transitive closure of the relation directly uses in $\mathcal{T}$. $\mathcal{T}$ is cyclic iff there is $A$ such that $A$ uses $A$ in $\mathcal{T}$.

Analogously, a fuzzy KB is said to be acyclic when its TBox is acyclic.

In the crisp case, acyclic TBoxes can be eliminated through an expansion process [15] which only takes a finite number of steps. The fact that this process can create an exponential growth of the KB has motivated the introduction of the lazy expansion optimization technique [3, very useful in practice.

In the fuzzy case, acyclic TBoxes cannot be completely removed in general due to the presence of degrees $\alpha$, and we need to require some additional property in order to do so. We say that a fuzzy TBox is unfoldable if it is an acyclic TBox which only contains axioms of the form $\langle A \sqsubseteq C \geq 1\rangle$, and $A \equiv C$ (that is, an acyclic TBox where every fuzzy concept inclusion axiom is of the form $\langle A \sqsubseteq C \geq \alpha\rangle$ with $\alpha=1$ ) (cf. [19, Section 3.3]). Analogously, a fuzzy KB is said to be unfoldable when its TBox is unfoldable.

In case we allow to use truth constants $\alpha$ as concept constructors, then it is trivially true for all left-continuous t-norms that $\langle C \sqsubseteq D \geq \alpha\rangle$ is equisatisfiable

${ }^{6}$ In particular this forbids concept $\top$ on the left hand side of axioms. 
with $\langle C \sqcap \alpha \sqsubseteq D \geq 1\rangle$. Next, we exploit a similar idea to show that, in the particular case of Łukasiewicz, unfoldable fuzzy TBoxes can be converted into acyclic ones, even without having truth constants $\alpha$ as concept constructors. The idea is that all axioms of the form $\langle A \sqsubseteq C \geq \alpha\rangle$ can be converted into the form $\left\langle A \sqsubseteq C^{\prime} \geq 1\right\rangle$. For example, $\left\langle A \sqsubseteq C \geq \frac{2}{3}\right\rangle$ can be converted into the form

$$
\left\langle A \sqsubseteq C \sqcup\left(\left(A^{\prime} \sqcap A^{\prime}\right) \sqcap \neg\left(A^{\prime} \sqcap A^{\prime} \sqcap A^{\prime}\right)\right) \geq 1\right\rangle,
$$

where $A^{\prime}$ is a new atomic concept; the reason why this is so is because the concept $\left(A^{\prime} \sqcap A^{\prime}\right) \sqcap \neg\left(A^{\prime} \sqcap A^{\prime} \sqcap A^{\prime}\right)$ always takes values onto the interval $\left[0, \frac{1}{3}\right]$. In the following proof we develop this idea.

Lemma 4.1 (Eukasiewicz Case) There is an algorithm that converts every acyclic fuzzy $K B \mathcal{K}=\langle\mathcal{A}, \mathcal{T}\rangle$ into an unfoldable fuzzy $K B \mathcal{K}^{\prime}=\left\langle\mathcal{A}, \mathcal{T}^{\prime}\right\rangle$ in such a way that

$$
\mathcal{K} \text { is satisfiable iff } \quad \mathcal{K}^{\prime} \text { is satisfiable. }
$$

The algorithm also preserves satisfiability in a witnessed model, and the same for satisfiability in a finite model.

Proof: Let us consider an axiom of the form $\langle A \sqsubseteq C \geq \alpha\rangle$. By the constructive version of McNaughton's Theorem (see [1]) it is obvious that there is an algorithm that for every rational $\alpha$ generates a propositional concept $\tau_{\alpha}\left(A^{\prime}\right)$ using only one atomic concept $A^{\prime}$ and such that

P1: $\tau_{\alpha}\left(A^{\prime}\right)$ only takes values in $[0,1-\alpha]$,

P2: $\tau_{\alpha}\left(A^{\prime}\right)$ takes value $1-\alpha$ when $A^{\prime}$ takes value $\alpha$.

To finish the proof it is enough to see that for every KB $\mathcal{K}$, it holds that

- $\mathcal{K} \cup\{\langle A \sqsubseteq C \geq \alpha\rangle\}$ is satisfiable, iff

- $\mathcal{K} \cup\left\{\left\langle A \sqsubseteq C \sqcup \tau_{\alpha}\left(A^{\prime}\right) \geq 1\right\rangle\right\}$ is satisfiable (where $A^{\prime}$ is a new atomic concept).

The upwards implication is a consequence of P1. On the other hand, the downwards implication is a consequence of P2 because we can extend any interpretation $\mathcal{I}$ to encompass the new atomic concept $A^{\prime}$ defining for every individual $x, A^{\mathcal{I}}(x)=\alpha$. It is also obvious that this way of extending the interpretation preserves the witnessed property (because $A^{\prime}$ takes the same value in all individuals) and the finiteness.

Q.E.D.

Next we prove, using ideas from [19, that fuzzy unfoldable TBoxes can be eliminated through a finite expansion process when the interpretation of $\sqcap$ is a continuous t-norm. Our proof of this result does not seem to work for satisfiability in a witnessed model; but we know how to deal with this case as long as the minimum constructor $\min \{C, D\}$ is definable in our framework. We remind the reader that in the case of continuous t-norms the constructor $\min \{C, D\}$ is definable, using an R-implication constructor $\rightarrow$, by $C \sqcap(C \rightarrow D)$; 
hence, the Zadeh, Łukasiewicz (note that in Łukasiewicz logic, $C \rightarrow D$ coincides with $\neg C \sqcup D$ ) and Gödel families given in Table 1 satisfy the assumption in the second item of the following result.

Lemma 4.2 (Assuming $\otimes$ is continuous) Let $\mathcal{K}$ be a fuzzy $K B$ and let $C, D$ be two concepts.

1. The following statements are equivalent:

- $\mathcal{K} \cup\{\langle C \sqsubseteq D \geq 1\rangle\}$ is satisfiable,

- $\mathcal{K} \cup\{C \equiv A \sqcap D\}$, where $A$ is a new atomic concept, is satisfiable.

This equivalence also holds when we consider satisfiability in a finite model.

2. (Assuming the constructor min is definable) The following statements are equivalent:

- $\mathcal{K} \cup\{\langle C \sqsubseteq D \geq 1\rangle\}$ is satisfiable,

- $\mathcal{K} \cup\{C \equiv \min \{A, D\}\}$, where $A$ is a new atomic concept, is satisfiable.

This equivalence also holds when we consider satisfiability in a finite model, and the same for satisfiability in a witnessed model.

Proof: Let us consider the first item. One direction is a consequence of the fact that t-norms satisfy $x \otimes y \leq y$. For the other, let us point out that continuous t-norms are divisible (see [11, Lemma 2.1.10]) in the sense that for every $x, y \in[0,1]$ there is an element $a$ such that $a \otimes x=\min \{x, y\}$; this element $a$ can be defined as $\sup \{z \in[0,1]: x \otimes z \leq y\}$ and is commonly denoted $x \Rightarrow y$. Hence, we can extend any interpretation $\mathcal{I}$ to encompass the new atomic concept $A$ defining $A^{\mathcal{I}}(x):=D^{\mathcal{I}}(x) \Rightarrow C^{\mathcal{I}}(x)$.

One direction of the second item follows from the fact that $\min \{x, y\} \leq y$. The other is proved extending any interpretation $\mathcal{I}$ to encompass the new atomic concept $A$ defining $A^{\mathcal{I}}(x):=C^{\mathcal{I}}(x)$.

Q.E.D.

\section{Lemma 4.3 (Assuming $\otimes$ is continuous)}

1. There is an algorithm that converts every unfoldable fuzzy $K B \mathcal{K}=\langle\mathcal{A}, \mathcal{T}\rangle$ into a fuzzy $A B o x \mathcal{A}^{\star}$ in such a way that

$$
\mathcal{K} \text { is satisfiable } \quad \text { iff } \quad \mathcal{A}^{\star} \text { is satisfiable. }
$$

The algorithm also preserves satisfiability in a finite model.

2. (Assuming the constructor min is definable) There is an algorithm like in the previous item which additionally also preserves satisfiability in a witnessed model. 
Proof: We give the proof of the first item. The second one is proved analogously but using the second item in Lemma 4.2

Let us assume that $\mathcal{K}=\langle\mathcal{A}, \mathcal{T}\rangle$ is an unfoldable fuzzy $\mathrm{KB}$. We know that all axioms in $\mathcal{T}$ are either of the form $\langle A \sqsubseteq C \geq 1\rangle$ or $A \equiv C$, where $A$ is an atomic concept. The algorithm consists on two steps. In the first step, we replace all axioms of the form $\langle A \sqsubseteq C \geq 1\rangle$ with $A \equiv A^{\prime} \sqcap C$ (with $A^{\prime}$ a new atomic concept). Once this is done we know that all axioms in the new TBox $\mathcal{K}^{\prime}$ are of the form $A \equiv C$. In the second step of the algorithm, we consider the ABox $\mathcal{A}$ and replace, for every axiom $A \equiv C$, all occurrences of $A$ with $C$. We will refer by $\mathcal{A}^{\star}$ to the output of this process.

Now let us check that $\mathcal{K}$ is satisfiable iff $\mathcal{A}^{\star}$ is satisfiable. The first item in Lemma 4.2 takes care of the first step of the algorithm, that is, $\mathcal{K}$ is satisfiable iff $\mathcal{K}^{\prime}$ is satisfiable. Next we prove that $\mathcal{K}^{\prime}$ is satisfiable iff $\mathcal{A}^{\star}$ is satisfiable. The rightwards direction is trivial. For the other, it is enough to notice that since the axioms in $\mathcal{K}^{\prime}$ are of the form $A \equiv C$ with $A$ an atomic concept we can redefine, for every individual $a$ appearing in the $\operatorname{ABox} \mathcal{A}^{\star}, A^{\mathcal{I}}\left(a^{\mathcal{I}}\right)$ as the value $C^{\mathcal{I}}\left(a^{\mathcal{I}}\right)$.

Q.E.D.

Next theorem is formulated only for Łukasiewicz in order to use the decidability result in [12, Corollary 1] for our framework. We include t-conorm as a concept constructor as opposed to [12, but Łukasiewicz t-conorm is clearly definable from Łukasiewicz t-norm and negation. Extensions of [12, Corollary 1] adding a t-norm constructor would be needed in order to deal with all t-norm logics considered in this paper, and not only Łukasiewicz.

\section{Theorem 4.4 (Łukasiewicz Case)}

1. For every fuzzy $K B \mathcal{K}=\langle\mathcal{A}, \mathcal{T}\rangle$ such that $\mathcal{T}$ is acyclic, the satisfiability in a finite model under the fuzzy $\mathcal{A L C}$ coincides with the satisfiability.

2. There is an algorithm for checking the satisfiability of acyclic KBs.

Proof: By Lemma 4.1 and Lemma 4.3 it is possible to remove the TBox. Then, we use the known results [12, Theorem 8 and Corollary 1] that in fuzzy $\mathcal{A L C}$ ABoxes, satisfiability in a witnessed model and satisfiability in a finite model coincide and are decidable problems. Note that [12] does not consider a t-conorm in the language, but it can defined from Eukasiewicz negation and t-norm. The proof finishes using the result in [13] which tells us that for Eukasiewicz, satisfiability coincides with satisfiability in a witnessed model. Q.E.D.

On the other hand, the second remark concerns the fact there are several reasoning algorithms in the literature for Łukasiewicz [7, 21, 22, Product [6], or any left continuous t-norm fuzzy DLs [ 8 , 18]) that claim to support GCIs. These algorithms restrict themselves to witnessed models. Unfortunately, these papers are implicitly assuming that the logic satisfies FMP. By Theorems 3.3 and 3.7 we have shown that this assumption cannot be done, so these algorithms do not work correctly for arbitrary fuzzy KBs (even if the semantics of GCIs is defined using Zadeh's set inclusion, as it happens in [18]). For instance, 
these algorithms are not able to provide a correct model for $\mathcal{K}_{2}$. Once these algorithms generate a tableau, it is unknown how to build an infinite model starting from it.

By the previous results, the cited algorithms are correct if we add some additional restrictions:

- 7, 21, 22, consider the fuzzy DL $\mathcal{A L C}$ under Eukasiewcz fuzzy logic. According to Theorem 4.4, the algorithms are correct in case we only consider acyclic KBs.

- 6] consider the fuzzy DL $\mathcal{A L C}$ under Product fuzzy logic. By Lemma 4.3 the algorithm is correct if we only consider unfoldable KBs.

- 8 provides reasoning algorithms for the fuzzy DLs $\mathcal{A L C}$ defined by families of fuzzy operators corresponding to a left-continuous t-norm extended with an involutive negation 7. This work restricts itself to acyclic KBs, so for instance $\mathcal{K}_{2}$ cannot be represented in the logic. According to Theorem 4.4, the algorithm is correct for Eukasiewicz logic. By Lemma 4.3, the algorithm is also correct for unfoldable KBs. The correctness of the algorithm for acyclic KBs any fuzzy logic different from Łukasiewicz remains a conjecture.

- 18 provides a reasoning algorithm for the fuzzy DLs $\mathcal{S I}$ defined by families of fuzzy operators corresponding to a left-continuous t-norm. Again, the algorithm is correct if we restrict to unfoldable KBs (Lemma 4.3), or to acyclic KBs and Łukasiewicz fuzzy logic (Theorem 4.4).

It is a matter of future research to determine the decidability of fuzzy $\mathcal{A L C}$ and sublanguages with an unrestricted TBox under Lukasiewicz and Product fuzzy logics.

\section{Acknowledgement}

We would like to thank to the anonymous referees for their very valuable comments on an earlier version of this paper. Special acknowledgements are due to Marco Cerami, Francesc Esteva and Àngel García-Cerdaña for their comments on a preliminary version.

\section{Funding}

Fernando Bobillo has been partially funded by the Spanish Ministry of Science and Technology (project TIN2009-14538-C02-01). Félix Bou thanks Euro-

\footnotetext{
${ }^{7}$ Here the fuzzy logic corresponding to a left continuous t-norm $\otimes$ is understood as based on the connectives given by the t-norm $\otimes$, its residuum, Eukasiewicz negation and the dual t-conorm of $\otimes$. This is quite different to the logic of left-continuous t-norms as used in [10, 11$]$ 12 , and closer (except for the use of truth constants as concept constructors) to the framework in 9 .
} 
cores (LOMOREVI Eurocores Project FP006/FFI2008-03126-E/FILO), Spanish Ministry of Education and Science (project MULOG2 TIN2007-68005-C0401), and Catalan Government (2009SGR-1433).

\section{References}

[1] Stefano Aguzzoli. The complexity of McNaughton functions of one variable. Advances in Applied Mathematics, 21(1):58-77, 1998.

[2] Franz Baader, Diego Calvanese, Deborah McGuinness, Daniele Nardi, Peter F. Patel-Schneider. The Description Logic Handbook: Theory, Implementation, and Applications. Cambridge University Press, 2003.

[3] Franz Baader, Enrico Franconi, Bernhard Hollunder, Bernhard Nebel, Hans-Jürgen Profitlich. An empirical analysis of optimization techniques for terminological representation systems or: Making KRIS get a move on. Applied Artificial Intelligence, 4:109-132, 1994.

[4] Franz Baader, Ian Horrocks, Ulrike Sattler. Description logics as ontology languages for the semantic web. In Dieter Hutter and Werner Stephan, editors, Mechanizing Mathematical Reasoning: Essays in Honor of Jörg H. Siekmann on the Occasion of His 60th Birthday, volume 2605 of Lecture Notes in Artificial Intelligence, p. 228-248. Springer-Verlag, 2005.

[5] Patrick Blackburn, Maarten de Rijke, Yde Venema. Modal Logic. Cambridge University Press, 2001.

[6] Fernando Bobillo, Umberto Straccia. A fuzzy description logic with product t-norm. In Proceedings of the 16th IEEE International Conference on Fuzzy Systems (FUZZ-IEEE 2007), p. 652-657. IEEE Press, 2007.

[7] Fernando Bobillo, Umberto Straccia. On qualified cardinality restrictions in fuzzy description logics under Eukasiewicz semantics. In Proceedings of the 12th International Conference of Information Processing and Management of Uncertainty in Knowledge-Based Systems (IPMU 2008), p. 1008-1015, 2008.

[8] Fernando Bobillo, Umberto Straccia. Fuzzy description logics with general t-norms and datatypes. Fuzzy Sets and Systems 160(23):3382-3402, 2009.

[9] Àngel García-Cerdaña, Eva Armengol, Francesc Esteva. Fuzzy description logics and t-norm based fuzzy logics. International Journal of Approximate Reasoning. In press.

[10] Petr Hájek. Metamathematics of Fuzzy Logic. Kluwer, 1998.

[11] Petr Hájek. Making fuzzy description logic more general. Fuzzy Sets and Systems, 154(1):1-15, 2005. 
[12] Petr Hájek. Computational complexity of t-norm based propositional fuzzy logics with rational truth constants Fuzzy Sets and Systems, 157(5):677$682,2006$.

[13] Petr Hájek. On witnessed models in fuzzy logic. Mathematical Logic Quarterly, 53(1):66-77, 2007.

[14] Thomas Lukasiewicz, Umberto Straccia. Managing uncertainty and vagueness in description logics for the semantic web. Journal of Web Semantics, 6(4):291-308, 2008.

[15] Bernhard Nebel. Reasoning and revision in hybrid representation systems. Volume 422 of Lecture Notes in Artificial Intelligence, p. 228-248. SpringerVerlag, 1990.

[16] Klaus Schild. A correspondence theory for terminological logics: Preliminary report. In Proceedings of the 12th International Joint Conference on Artificial Intelligence (IJCAI 1991), p. 466-471. Morgan Kaufmann, 1991.

[17] Manfred Schmidt-Schauß, Gert Smolka. Attributive concept descriptions with complements. Artificial Intelligence, 48(1):1-26, 1991.

[18] Giorgos Stoilos, Giorgos Stamou. A framework for reasoning with expressive continuous fuzzy description logics. In Proceedings of the 22nd International Workshop on Description Logics (DL 2009). CEUR Workshop Proceedings, 2009.

[19] Umberto Straccia. Reasoning within fuzzy description logics. Journal of Artificial Intelligence Research, 14:137-166, 2001.

[20] Umberto Straccia. A fuzzy description logic for the semantic web. In Elie Sanchez, editor, Fuzzy Logic and the Semantic Web, volume 1 of Capturing Intelligence, p. 73-90. Elsevier Science, 2006.

[21] Umberto Straccia, Fernando Bobillo. Mixed integer programming, general concept inclusions and fuzzy description logics. In Proceedings of the 5th Conference of the European Society for Fuzzy Logic and Technology (EUSFLAT 2007), volume 2, p. 213-220, 2007.

[22] Umberto Straccia, Fernando Bobillo. Mixed integer programming, general concept inclusions and fuzzy description logics. Mathware $\&$ Soft Computing, 14(3):247-259, 2007.

[23] Lotfi A. Zadeh. Fuzzy sets. Information and Control, 8:338-353, 1965. 